\title{
Dishevelled effect
}

DOI:

10.1038/nrn1940

URLs

Pull quote

It will be interesting to determine how PCP proteins co-evolve to regulate diverse developmental processes across many species.

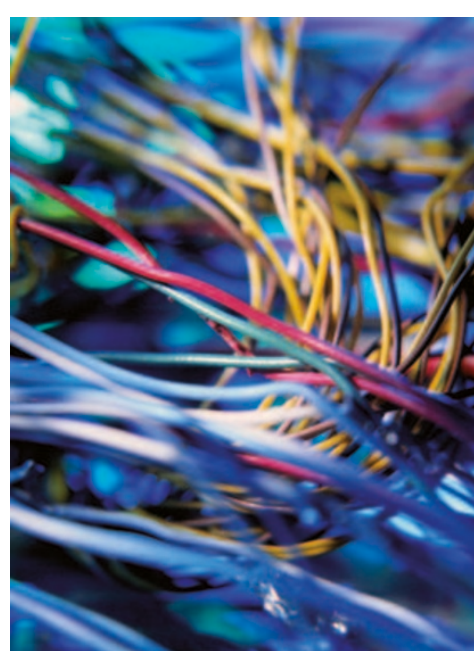

Although the planar cell polarity (PCP) pathways, which regulate cell orientation within the plane of an epithelium, are conserved throughout evolution, they mediate distinct developmental processes in different organisms. In a study published in Development, Jianbo Wang and colleagues uncover a mammalian PCP pathway that regulates concomitant lengthening and narrowing of the neural plates - a morphogenic process defined as convergent extension.
In the fruitfly, in which PCP was first described, activated Frizzled receptors trigger the redistribution of core PCP proteins, including Dishevelled (DSH) and Flamingo; these proteins then specify the orientation of cells and their associated bristles. Wang et al. showed previously that, in the mouse cochlea, the PCP pathways regulate both cell polarity and polarized extension. In this new study, mice lacking the mammalian Dsh homologue Dvl2 had severe defects in neural tube closure - a phenotype that was not seen in Dvl1or Dvl3-deficient mice - suggesting that $D v l 2$ is the most important mammalian $D v l$ gene for regulating this developmental process.

To identify the functional domain of DVL2 that is important for controlling convergent extension, the researchers introduced into the mouse various $D v l 2$ transgenes with different domain deletions. They found that the carboxy-terminal DEP domain, which is solely required for the function of its homologues in PCP in flies and convergent extension in Xenopus, was crucial for correct membrane localization of DVL2 - a pre-requisite for its involvement in convergent extension - as well as its function in neurulation. Furthermore, a $D v l 2$ mutant carrying a point mutation identical to the Dsh 1 allele that abolishes the PCP pathway in the fly was no longer able to regulate convergent extension or the polarity of cochlea sensory hair cells.

This study has shed fresh light on the remarkable similarities between the PCP pathway in the fly and convergent extension in the mouse. It will be interesting to determine how PCP proteins co-evolve to regulate diverse developmental processes across many species.

Jane Qiu

ORIGINAL RESEARCH PAPER Wang, J. et al. Dishevelled genes mediate a conserved mammalian PCP pathway to regulate convergent extension during neurulation. Development 133 , 1767-1778 (2006)

FURTHER READING Wallingford, J. B. \& Habas, R

The developmental biology of Dishevelled: an enigmatic protein governing cell fate and cell polarity. Development 132, 4421-4436 (2005) WEB SITE

Wynshaw-Boris's laboratory: http://medicine. ucsd.edu/faculty/Wynshaw-Boris/ 